\title{
Inclusive searches for squarks and gluinos in final states with leptons with the ATLAS detector
}

\section{Ximo Poveda, on behalf of the ATLAS Collaboration}

European Laboratory for Particle Physics, CERN

E-mail: Joaquin.Poveda@cern.ch

Despite the absence of experimental evidence, supersymmetry remains one of the best motivated and studied Standard Model extensions. These proceedings summarise recent results from the ATLAS experiment at the LHC on inclusive searches for supersymmetric squarks and gluinos, including third generation squarks produced in the decay of gluinos. The searches involve final states containing jets and one or more leptons, and were performed with $p p$ collisions at a centreof-mass energy of $13 \mathrm{TeV}$.

EPS-HEP 2017, European Physical Society conference on High Energy Physics 5-12 July 2017

Venice, Italy 


\section{Introduction}

Supersymmetry (SUSY) is one of the most studied theoretical frameworks to extend the Standard Model (SM) beyond the electroweak scale; a general review can be found in Ref. [1]. It predicts a new bosonic (fermionic) partner for each fundamental SM fermion (boson), as well as two Higgs doublets. The SUSY partners of the Higgs and electroweak gauge bosons mix to form the mass eigenstates known as charginos $\left(\tilde{\chi}_{l}^{ \pm}, l=1,2\right.$, ordered by increasing mass) and neutralinos $\left(\tilde{\chi}_{m}^{0}, m=1, \ldots, 4\right.$, ordered by increasing mass). In SUSY models, leptons can appear in decay chains involving $W / Z$ bosons or sleptons, and in the decay of neutralinos (such as $\tilde{\chi}_{1}^{0} \rightarrow \ell^{+} \ell^{-} v$ ) in some $R$-parity violating models. Leptonic final states provide robust experimental handles, which are particularly useful to discriminate a SUSY signal from the background: leptonically decaying $Z$ bosons, kinematic edges in the dilepton invariant mass (which could also be used to measure SUSY particle masses), production of same-sign dilepton pairs (in gluino production or long decay chains), etc. These proceedings present recent searches for squarks $(\tilde{q})$ and gluinos $(\tilde{g})$ using $p p$ collision data at $\sqrt{s}=13 \mathrm{TeV}$ collected by the ATLAS experiment [2] at the LHC in singlelepton [3], opposite-sign (OS) dilepton [4], and same-sign dilepton or trilepton final states [5].

\section{Search in single lepton final states}

The first analysis [3] targets SUSY models involving W/Z bosons, appearing in either short or long decay chains. They are probed with signal regions (SRs) with exactly one lepton, large missing transverse momentum $\left(E_{\mathrm{T}}^{\text {miss }}\right)$, and either $\geq 2$ jets, $\geq 4$ jets, $\geq 6$ jets or $\geq 9$ jets. The sensitivity of the SRs is enhanced relying on additional variables such as the effective mass $m_{\text {eff }}$ (scalar sum of the $p_{\mathrm{T}}$ of the leptons and jets plus the $E_{\mathrm{T}}^{\text {miss }}$ ) or the number of $b$-jets in the event. Control regions (CRs) are used to estimate the main backgrounds, requiring at least one $b$-jet for the estimation of the top-quark backgrounds, and a $b$-jet veto for the estimation of the $W+$ jets background.

As shown in Fig. 1, no significant excess is observed in the SRs, with the largest excess being in the 2-jet $b$-veto SRs. The results are used to derive exclusion limits, as illustrated in Fig. 2, which exclude gluino (squark) masses of 2.1 (1.2) TeV for the case of a simplified model [6] featuring $\tilde{g} \tilde{g}(\tilde{q} \tilde{q})$ production with $\tilde{g} \rightarrow q q W \tilde{\chi}_{1}^{0}\left(\tilde{q} \rightarrow q W \tilde{\chi}_{1}^{0}\right)$. Limits are also computed for varying mass hierarchies of the particles included in the model.

\section{Search in opposite-sign dilepton final states}

The second analysis [4] contains two different searches which rely on the OS + jets $+E_{\mathrm{T}}^{\text {miss }}$ topology: leptonically decaying on-shell $Z$ bosons $(Z \rightarrow \ell \ell)$ produced in the decay chain of squarks and gluinos (leading to an excess of events at $m_{\ell \ell} \sim m_{Z}$ ), and cascade decays like $\tilde{\chi}_{2}^{0} \rightarrow \ell \tilde{\ell} \rightarrow \ell \ell \tilde{\chi}_{1}^{0}$ (leading to an excess with triangular "edge" shape in the $m_{\ell \ell}$ distribution). The on-shell $Z$ search showed a $3 \sigma$ excess in Run-1 [7], and its SR has therefore been unchanged since then. The edge search uses SRs for low/medium/high $H_{\mathrm{T}}$ (scalar sum of the $p_{\mathrm{T}}$ of all jets in the event), divided in 24 overlapping $m_{\ell \ell}$ windows. In both cases, the flavor symmetric background $(t \bar{t}, W W)$ is estimated from $e \mu$ data, and the $Z+$ jets background is estimated from $\gamma+$ jets data. 


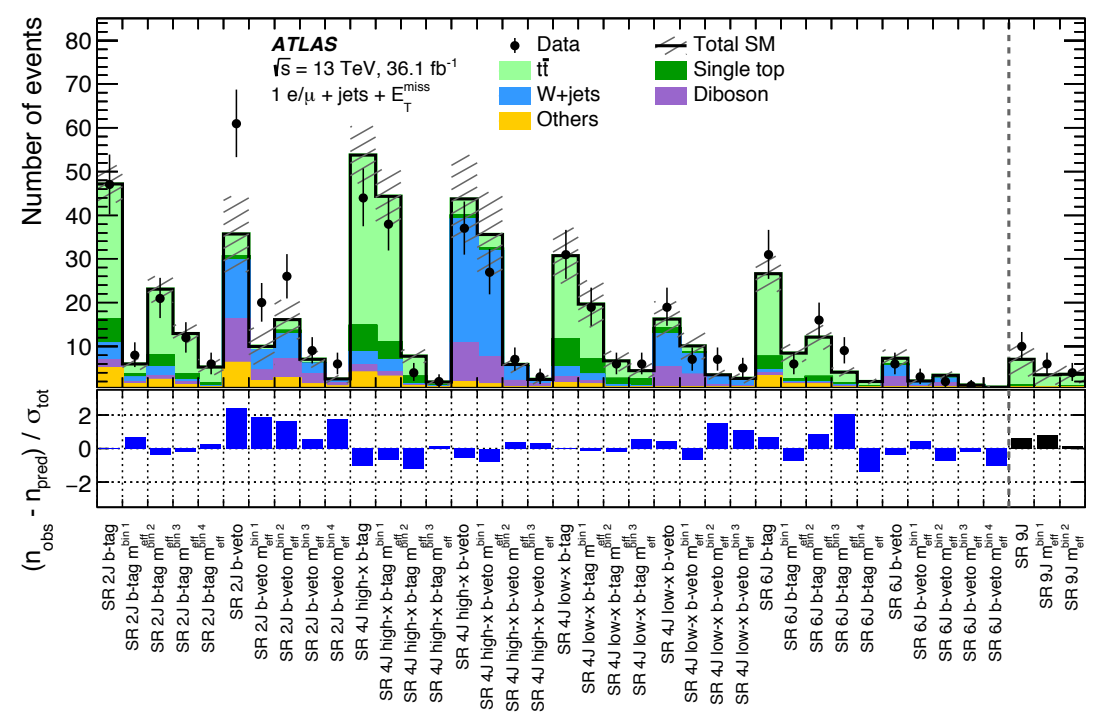

Figure 1: Comparison of the observed and expected event yields in the single-lepton signal regions [3], which are named in the form "SR $N \mathrm{~J}[\mathrm{X}-\mathrm{x}][\mathrm{b}-T][M]$ ", where $N$ indicates the number of jets required, $X$ indicates whether the region targets large/low value of the ratio of mass differences between $\tilde{\chi}_{1}^{ \pm}-\tilde{\chi}_{1}^{0}$ and $\tilde{q} / \tilde{g}-\tilde{\chi}_{1}^{0}, T$ indicates whether $b$-jets or a $b$-veto is required, and $M$ indicates the $m_{\text {eff }}$ requirements employed.
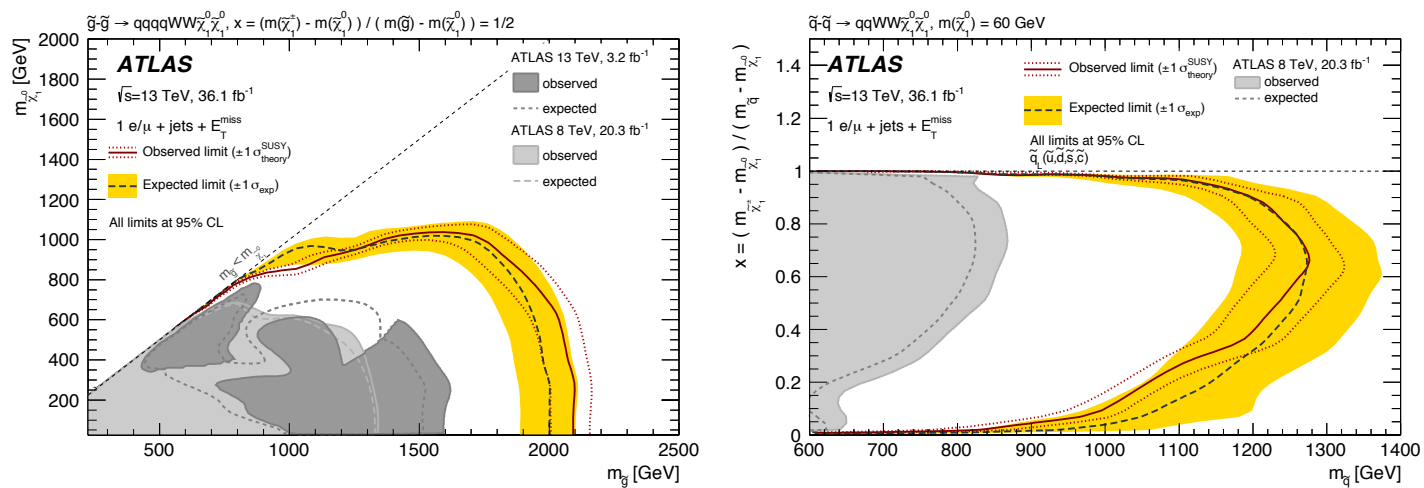

Figure 2: Exclusion contours in simplified models featuring gluino pair production and $\tilde{g} \rightarrow q q W \tilde{\chi}_{1}^{0}$ decay as a function of the gluino and neutralino masses (left), and featuring squark pair production and $\tilde{q} \rightarrow q W \tilde{\chi}_{1}^{0}$ decay as a function of the squark mass and the differences in mass between the ratio of the mass differences between $\tilde{\chi}_{1}^{ \pm}-\tilde{\chi}_{1}^{0}$ and $\tilde{q}-\tilde{\chi}_{1}^{0}[3]$.

The results of these searches are shown in Fig. 3, with no significant excess observed and the largest deviation from the SM prediction corresponding to a $1.7 \sigma$ local significance from the edge search in the SR at high $H_{\mathrm{T}}$ and $12<m_{\ell \ell}<101 \mathrm{GeV}$.

Some of the interpretations in terms of exclusion limits are shown in Fig. 4, excluding gluino masses up to $1.3 \mathrm{TeV}$ in simplified models featuring $\tilde{\chi}_{2}^{0} \rightarrow Z \tilde{\chi}_{1}^{0}$ decays. Interpretations in simplified models with $\tilde{g} \rightarrow q q \tilde{\chi}_{2}^{0}, \tilde{\chi}_{2}^{0} \rightarrow \ell \tilde{\ell} \rightarrow \ell \ell \tilde{\chi}_{1}^{0}$ exclude gluino masses up to $1.8 \mathrm{TeV}$ and neutralino masses of up to $900 \mathrm{GeV}$. 

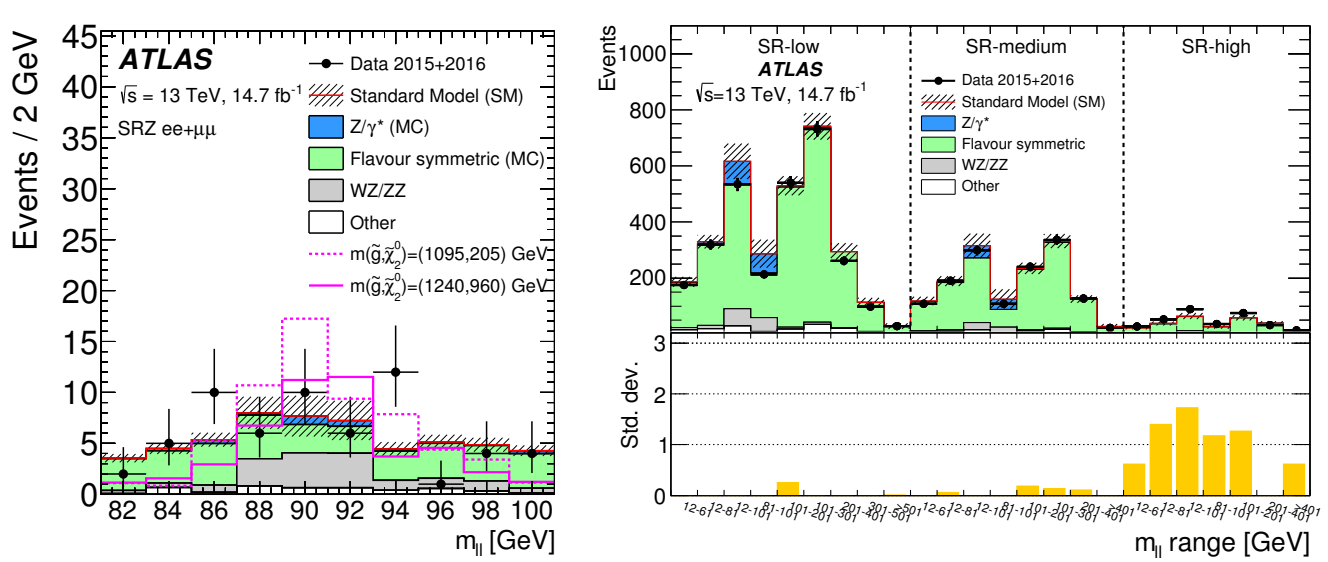

Figure 3: Results from the opposite-sign dilepton analysis [4]: dilepton invariant mass distribution for the on-shell $Z$ signal region (left) and comparison of the observed and expected event yields in the edge signal regions (right).
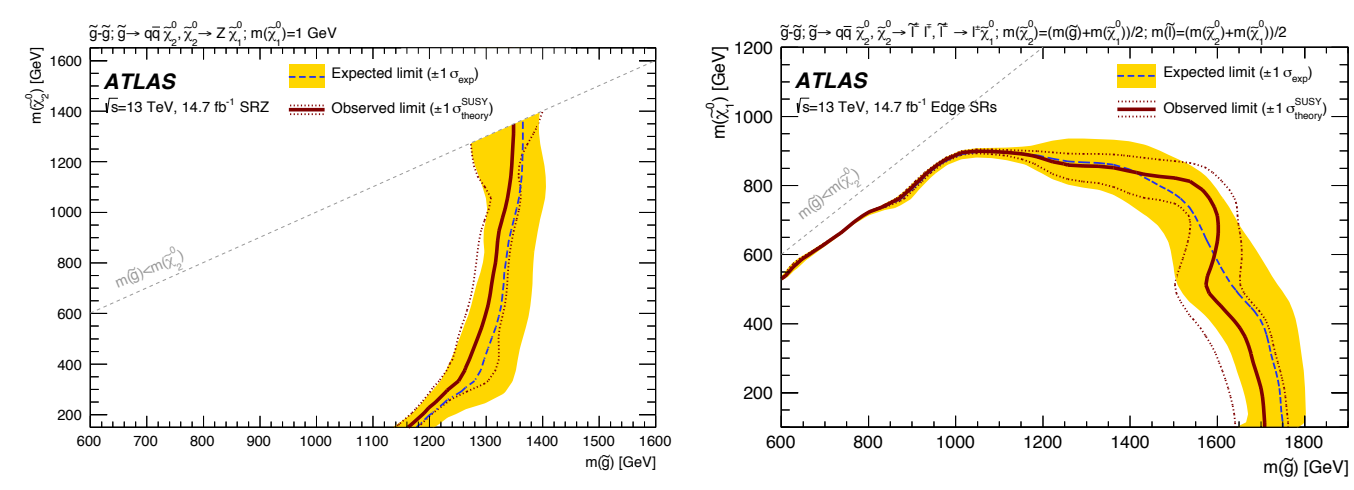

Figure 4: Exclusion contours in simplified models featuring gluino pair production and $\tilde{g} \rightarrow q q \tilde{\chi}_{2}^{0}$ decays, with either $\tilde{\chi}_{2}^{0} \rightarrow Z \tilde{\chi}_{1}^{0}$ (left) or $\tilde{\chi}_{2}^{0} \rightarrow \ell \tilde{\ell} \rightarrow \ell \ell \tilde{\chi}_{1}^{0}$ (right) [4].

\section{Search in same-sign dilepton and trilepton final states}

Finally, the third analysis [5] relies on final states containing same-sign (SS) dileptons or trilepton plus jets. This signature has broad sensitivity to many SUSY models involving gluinos or $W / Z / \tilde{\ell}$, both in $R$-parity conserving and $R$-parity violating SUSY scenarios. This analysis features looser lepton and jet kinematic requirements than other searches due to the background rejection from the SS requirement leading to better sensitivity to compressed scenarios. This is the only search exploring sbottom production with $\tilde{b} \rightarrow t \tilde{\chi}_{1}^{ \pm}$and $R$-parity violating right-handed d-squark $\left(\tilde{d}_{R}\right)$ production. Furthermore, this analysis exploits for the first time the signature of three leptons with the same electric charge $\left(\ell^{ \pm} \ell^{ \pm} \ell^{ \pm}\right)$.

Different signal regions are defined depending on the requirements applied on the number of jets and $b$-jets, as well as on $E_{\mathrm{T}}^{\text {miss }}$, an the results are shown in Fig. 5. No significant excess is observed, and some of the resulting exclusion limits are shown in Fig. 6. In $R$-parity conserving simplified models, limits exclude sbottom masses up to $700 \mathrm{GeV}$ and gluino masses up to 1.6- 


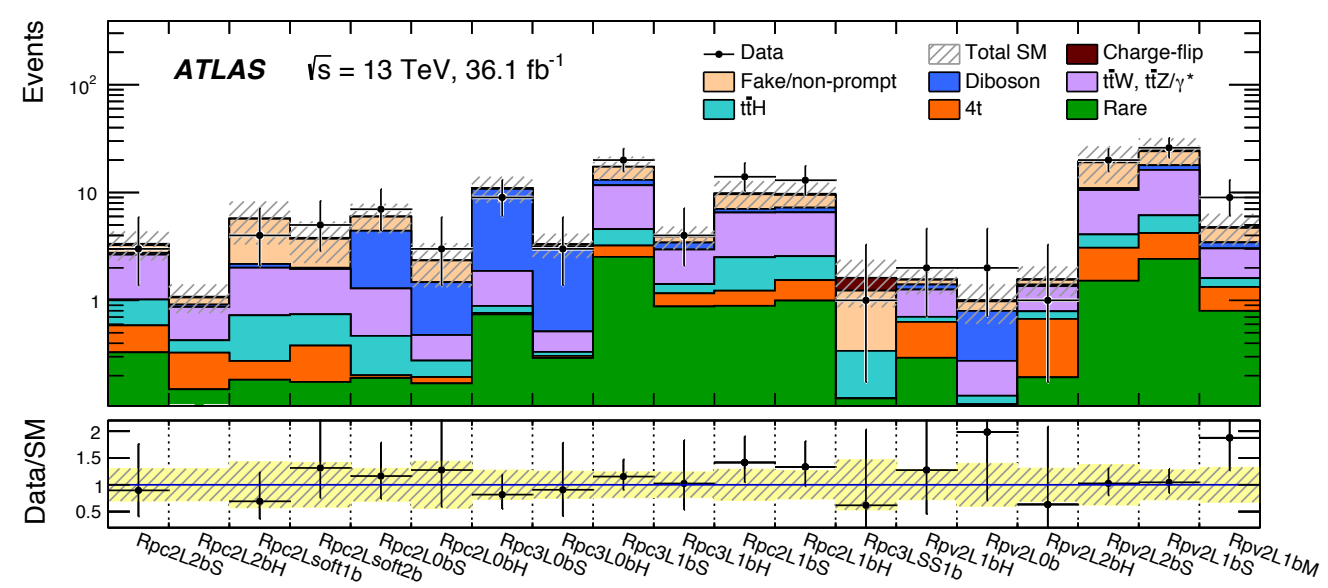

Figure 5: Comparison of the observed and expected event yields in the dilepton same-sign/trilepton signal regions [5], which are named in the form $S N \mathrm{~L} M \mathrm{~b} X$, where $S$ indicates if the region is targeting an $R$-parity conserving or violating model, $N$ indicates the number of leptons required, $M$ the number of $b$-jets required, and $X$ indicates the severity of the $E_{\mathrm{T}}^{\text {miss }}$ or $m_{\text {eff }}$ requirements (Soft, Medium or Hard).
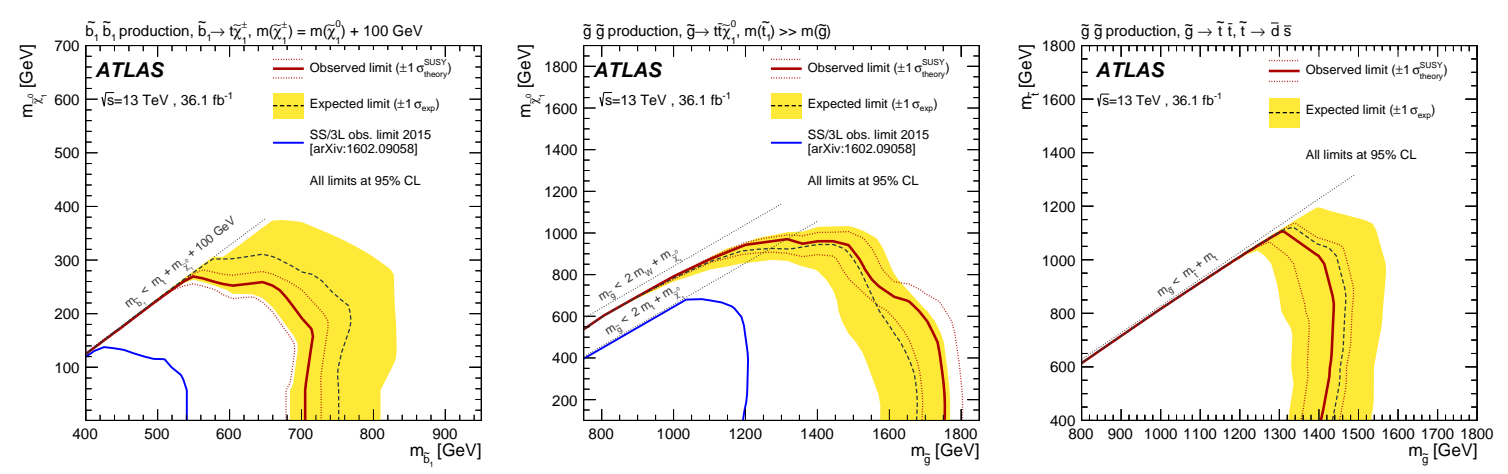

Figure 6: Exclusion contours in simplified models featuring sbottom pair production and $\tilde{b} \rightarrow t \tilde{\chi}_{1}^{ \pm}$decays (left), and guino pair production with either $\tilde{g} \rightarrow t \bar{t} \tilde{\chi}_{1}^{0}$ decays (centre) or $\tilde{g} \rightarrow \tilde{t} \bar{t}$ plus $\tilde{t} \rightarrow \bar{d} \bar{s} R$-parity violating decays (right) [5].

$1.8 \mathrm{TeV}$, with a very good improvement also for compressed scenarios, excluding neutralino masses of up to $\sim 900 \mathrm{GeV}$. In the considered $R$-parity violating simplified models, gluino masses of 1.4-1.8 $\mathrm{TeV}$ are excluded, with also very good coverage for compressed topologies, and exclusion limits of around $500 \mathrm{GeV}$ are obtained in models with $\tilde{d}_{R}$ pair production.

\section{Summary}

Signatures with leptons provide a powerful and versatile tool to search for squarks and gluinos at the LHC. These proceedings summarise recent searches in ATLAS using signatures with one to three leptons. With no excess observed, the current exclusion limits reach gluino masses of up to $2 \mathrm{TeV}$ with improved sensitivity at high neutralino masses and in compressed topologies compared to previous searches. 


\section{References}

[1] S. P. Martin, “A Supersymmetry primer,” Adv. Ser. Direct. High Energy Phys. 21 (2010) 1.

[2] ATLAS Collaboration, "The ATLAS Experiment at the CERN Large Hadron Collider," JINST 3 (2008) S08003.

[3] ATLAS Collaboration, "Search for squarks and gluinos in events with an isolated lepton, jets and missing transverse momentum at $\sqrt{s}=13 \mathrm{TeV}$ with the ATLAS detector", arXiv:1708.08232 [hep-ex].

[4] ATLAS Collaboration, "Search for new phenomena in events containing a same-flavour opposite-sign dilepton pair, jets, and large missing transverse momentum in $\sqrt{s}=13 \mathrm{TeV} p p$ collisions with the ATLAS detector", Eur. Phys. J. C (2017) 77: 144.

[5] ATLAS Collaboration, "Search for supersymmetry in final states with two same-sign or three leptons and jets using $36 \mathrm{fb}^{-1}$ of $\sqrt{s}=13 \mathrm{TeV} p p$ collision data with the ATLAS detector", arXiv:1706.03731 [hep-ex].

[6] D. Alves et al. [LHC New Physics Working Group], "Simplified Models for LHC New Physics Searches,” J. Phys. G 39 (2012) 105005.

[7] ATLAS Collaboration, "Search for supersymmetry in events containing a same-flavour opposite-sign dilepton pair, jets, and large missing transverse momentum in $\sqrt{s}=8 \mathrm{TeV}$ pp collisions with the ATLAS detector,” Eur. Phys. J. C 75 (2015) no.7, 318 Erratum: [Eur. Phys. J. C 75 (2015) no.10, 463]. 